Rev. Elev. Méd. vét. Pays trop., 1979, 32 (4) : 353-359

\title{
Enquête sur le parasitisme et la mortalité des veaux dans le Nord de la Côte-d'Ivoire : Observations préliminaires ${ }^{+}$
}

\author{
par G. S. MISHRA $\left(^{*}\right)$, E. CAMUS $\left(^{* *}\right)$, J. BELOT $\left({ }^{* *}\right)$ et A. E. N'DEPO $\left({ }^{* * *}\right)$ \\ RÉSUMÉ \\ Les enquêtes sur la mortalité des veaux, en milieu encadré, dans le Nord \\ de la Côte-d'Ivoire ont montré que son taux est de 11,9 p. 100. Elle intéresse \\ principalement les jeunes jusqu'à l'âge de 3 mois et se manifeste surtout en \\ début de saison sèche, de novembre à janvier. Au cours de cette enquête, qui a \\ duré 18 mois (janvier 1976-juın 1977), de nombreux prélèvements de fèces et de \\ sang ont été examinés, ce qui a permis d'établır que les causes principales des \\ fortes mortalités parfois observées sont par ordre d'importance les trypano- \\ somoses, la coccidiose, la strongyloidose et la strongylose alors que l'ascari- \\ diose occupe une très importante place dans la mortalité des jeunes en milieu \\ d'élevage traditionnel. L'existence de Theileria, Babesia, Anaplasma et de micro- \\ filarres a été mise en évidence mais il semble que ces parasites ne jouent pas \\ dans la mortalité des veaux un rôle nettement définı. De même la traite des \\ vaches, là où elle est pratiquée, ne paraît pas intervenir comme facteur déter- \\ minant de la mortalité des jeunes.
}

\section{INTRODUCTION}

Le développement de l'élevage dans le nord de la Côte-d'Ivoire se heurte à un certain nombre de facteurs limitants dont le moindre n'est pas la mortalité élevée qui se constate chez les jeunes animaux. C'est pour lutter contre que la Société d'Etat pour le Développement des Productions animales a mis en place, dans le nord du Pays une structure d'encadrement vétérinaire et zootechnique des éleveurs sédentaires, dont le troupeau est essentiellement constitué de taurins Baoulé et N'Dama, avec une tendance marquée au croisement Zébu-taurin dans un rayon d'environ $200 \mathrm{~km}$ de Korhogo.

$\left.{ }^{(}\right)$Travail exécuté dans le cadre du projet FAO/PNUD IVC/71/505 en collaboration avec la SODEPRA.

(*) FAO. Expert en Parasitologie, B. P. 1747, Abidjan. Adresse actuelle: FAO/PNUD. B. P. 345 Vientiane. Lao.

(**) Cellule d'Appui, SODEPRA, Korhogo.

(***) Expert associé en Parasitologie, Korhogo.

(****) Parasitologue, Bingerville.
Il s'agit d'exposer, dans cette étude préliminaire, l'incidence générale du parasitisme interne et externe sur la mortalité des veaux de ce " milieu encadré », le sujet devant être traité ultérieurement de façon approfondie, au fur et à mesure que les observations engrangées permettront d'en tirer des conclusions fixant de façon concrète la politique à suivre au mieux du but poursuivi.

Les résultats qui suivent ne concernent donc que ceux enregistrés dans le cadre de cet élevage "encadré "-qui tend à se répandre largement dans le milieu éleveur régional - et qui par rapport à l'élevage traditionnel se traduit par des actions diverses, dont les plus marquantes portent :

I. Sur les parcs de stabulations : qui sont plus vastes, mieux entretenus et plus propres de façon à offrir aux animaux, notamment aux jeunes produits, des conditions d'hygiène générale, de repos et de sécurité propres à favoriser leur épanouissement. 
II. Sur une action sanitaire en profondeur, diversifiée et soutenue qui se traduit par :

a) la vaccination à partir de 6 mois contre la peste et la péripneumonie bovines et contre le charbon symptomatique avant le début de chaque saison des pluies;

b) le déparasitage interne des veaux, qui, de 0 à 2 ans, reçoivent suivant le cas du Tétramisole ou du tartrate de Morantel en début et en fin de la saison des pluies.

Dans l'intervalle les veaux d'âge compris entre 15 jours et un mois et demi font l'objet chaque mois d'un traitement antiparasitaire approprié à la nature de leur parasitisme ;

c) leur déparasitage externe, qui vise surtout à les débarrasser des tiques très nombreuses dans la région considérée. Il s'effectue à l'aide de pulvérisateurs à dos et de produits organochlorés ou organophosphatés au rythme conseillé d'une fois par semaine en saison des pluies et une fois par mois en saison sèche.
Il convient de noter que ces jeunes veaux qui appartiennent à des races à haut degré de trypanotolérance ne font pratiquement jamais l'objet de traitements trypanocides, en dépit de la présence de nombreuses glossines vectrices de trypanosomes pathogènes variés. Cette absence de traitement a eu à la fois pour but de préciser l'impact des trypanosomoses locales sur la mortalité des jeunes veaux dans les parcs encadrés et d'étudier sur ces mêmes sujets l'installation et le développement de la trypanotolérance qui caractérise leur race ;

d) des soins individuels donnés aux animaux atteints de diarrhée (sulfamides en poudre) ou de plaies diverses (solutions ou pommades antiseptiques classiques).

III. Sur l'amélioration de l'alimentation des veaux : en leur assurant la totalité du lait de leur mère. Malheureusement, en dépit des mesures d'encadrement dont les troupeaux encadrés sont l'objet, cette mesure n'est vrai-

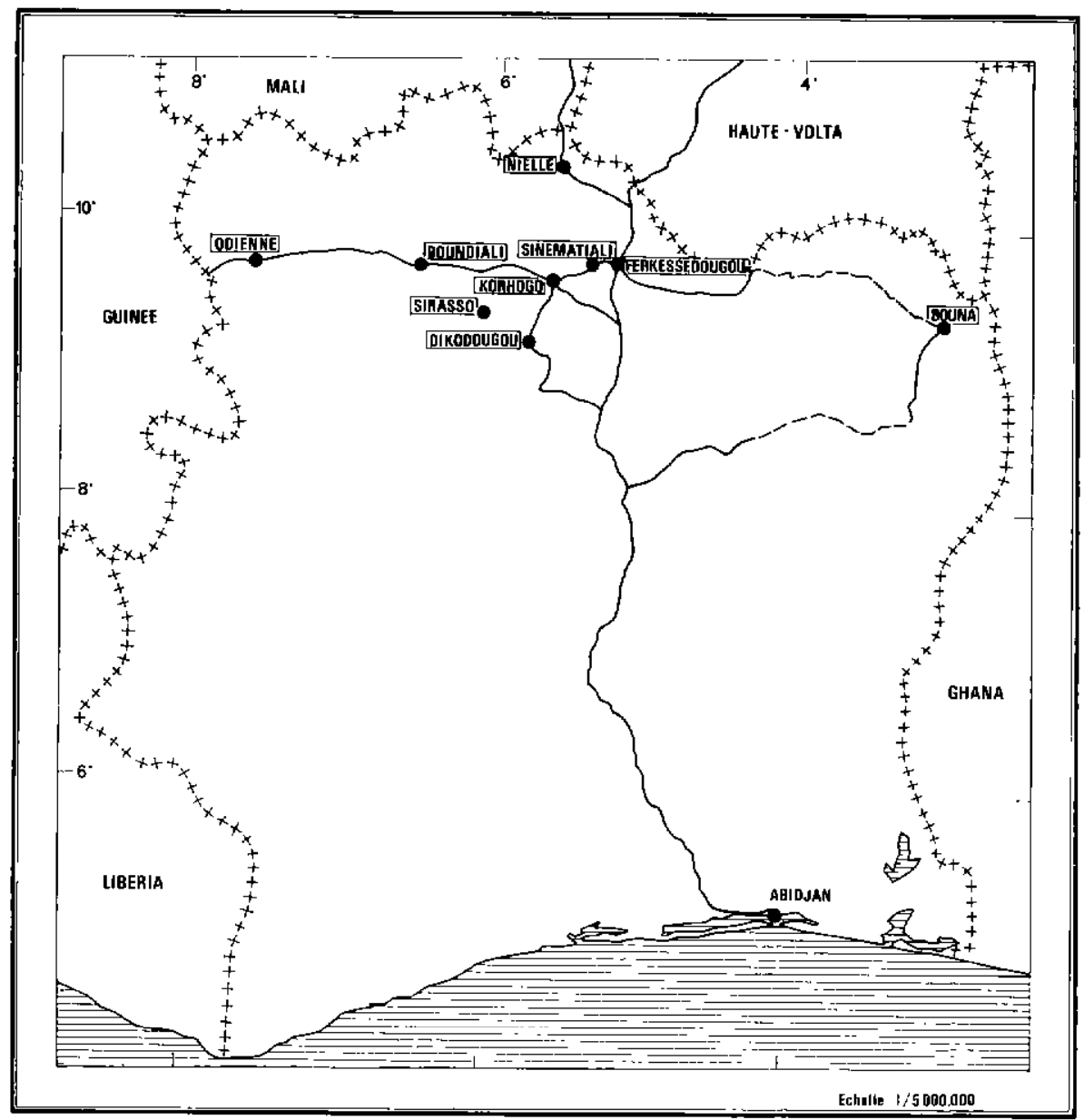


ment appliquée que durant les premières semaines de leur existence. Ensuite dans la plupart des cas, c'est-à-dire dans environ 80 p. 100 des parcs encadrés, la vache est traitée en partie et son lait utilisé ou commercialisé par son propriétaire (GODET, 1977). Dans ces conditions, les veaux sont amenés à consommer très tôt de l'herbe, bien que le sevrage n'intervienne souvent qu'après un an.

Ces observations qui ont recouvert le nord de la Côte-d'Ivoire, de janvier 1976 à juin 1977, soit pendant 18 mois, ont plus particulièrement intéressé les préfectures d'Odienné, Boundiali, Korhogo, Ferkéssédougou et Bouna.

\section{MATERIEL ET MÉTHODES}

a) Les enquêtes sont effectuées par sondages, soit dans deux parcs suivis régulièrement (6) soit de façon systématique dans les parcs où de fortes mortalités sont signalées (4).

b) Les taux de mortalité ont été calculés, par classe d'âge, pour chaque parc en comparant le nombre de naissances à celui des morts.

c) Les prélèvements effectués ont intéressé les fèces, le sang (frottis, gouttes épaisses, tubes capillaires à hématocrites) et les tiques. Dans ceux à forte mortalité, les enquêteurs se sont efforcés d'en situer les causes sans toutefois avoir la possibilité, pour des raisons de conjoncture, de pratiquer un nombre suffisant d'autopsies pour en tirer des conclusions concrétement valables.

En ce qui concerne les tiques, le but poursuivi a été d'en préciser le genre et d'étudier leur cycle saisonnier. A cet effet, mensuellement, dix veaux ont été choisis au hasard dans deux troupeaux et la totalité des tiques infestant chaque sujet a été récoltée. Conservées dans une solution d'alcool à 70 p. 100 dans des flacons individualisés, ces récoltes ont permis d'établir leur population par animal et par mois ce qui a permis d'établir un indice général d'infestation des animaux pour chaque période considérée.

\section{d) Analyses}

Les coproscopies ont été effectuées avec la cellule de Macmaster.

Les hématoscopies ont été effectuées par coloration au May Grūnwald Giemsa pour les frottis minces et les gouttes épaisses ; et l'hémato- crite mesurée avec recherche directe des parasites sous objectif en immersion suivant la méthode de Woo, 1970.

Les tiques ont été identifiées quant à leur genre suivant les données classiques en la matière.

\section{RÉSULTATS}

\section{a) Taux de mortalité}

Pour l'ensemble des troupeaux encadrés du nord de la Côte-d'Ivoire, le taux global de mortalité a été chez les jeunes de 12 p. 100 (trois mille cinq cent seize veaux de 0 à 1 an sont morts pour vingt-neuf mille cinq cent trentequatre naissances) pour les 18 mois d'observation.

Ce sont surtout les anjmaux de 0 à 3 mois qui ont payé le plus lourd tribut à cette mortalité, qui est surtout conséquente de novembre à février, c'est-à-dire en fin de saison des pluies et en début de saison sèche.

Ce taux global de 12 p. 100 recouvre des disparités régionales, parfois marquées, puisqu'il s'établit aux environs de 14 p. 100 pour les zones de Korhogo, Boundiali et Ferkéssédougou pour tomber à 8,5 p. 100 seulement dans les districts de Bouna, Odienné et Touba.

\section{b) Coproscopies}

Quatre cent soixante-dix échantillons de fèces de veaux d'âge, de sexe et de races différents ont été examinés, ce qui a permis de situer, de façon globale, le parasitisme du tube digestif à 61 p. 100 .

Avec par ordre d'importance: les strongles 42 p. 100 ; coccidies : 17 p. 100 ; ascaris : 6 p. 100 ; strongyloïdes : 4 p. 100 ; cestodes : 4 p. 100 , dont les espèces ont été identifiées, du fait de leur importance dans le choix des médicaments à utiliser pour les combattre.

Le taux d'infection des animaux par ces parasites varie avec l'âge des veaux, ainsi qu'il est précisé dans le tableau $n^{\circ} I$ et le graphique $n^{0} I$.

Il permet de noter que de 0 à 3 mois les coccidies, ascaris et strongyloïdes représentent respectivement un pourcentage d'infection de 23,18 et 12 p. 100 . A partir de 3 mois, le taux pour les coccidies évolue entre 15 et 21 p. 100 alors que les ascaris et les strongyloïdes disparaissent progressivement vers l'âge de 1 an et que les strongles augmentent graduellement jusqu'à cet âge. 
TABL, $N^{\circ} 1$-Pourcentage des veaux de différentes classes d'âges infestés par divers parasites

\begin{tabular}{|c|c|c|c|c|c|}
\hline \multirow{2}{*}{$\begin{array}{c}\text { Classes } \\
\text { d'âges } \\
\text { en mois }\end{array}$} & \multicolumn{3}{|c|}{ Pourcentage d'infestation des veaux par : } \\
\cline { 2 - 6 } & Strongle & Coccidie & Ascaris & Strongyloides & Cestode \\
\hline $0-3$ & 22 & 23 & 18 & 12 & 3 \\
$3-6$ & 45 & 21 & 5 & 5 & 7 \\
$6-9$ & 32 & 16 & 4 & 2 & 5 \\
$9-12$ & 56 & 12 & 2 & 1 & 6 \\
12 et plus & 37 & 15 & 0 & 0 & 6 \\
\hline
\end{tabular}

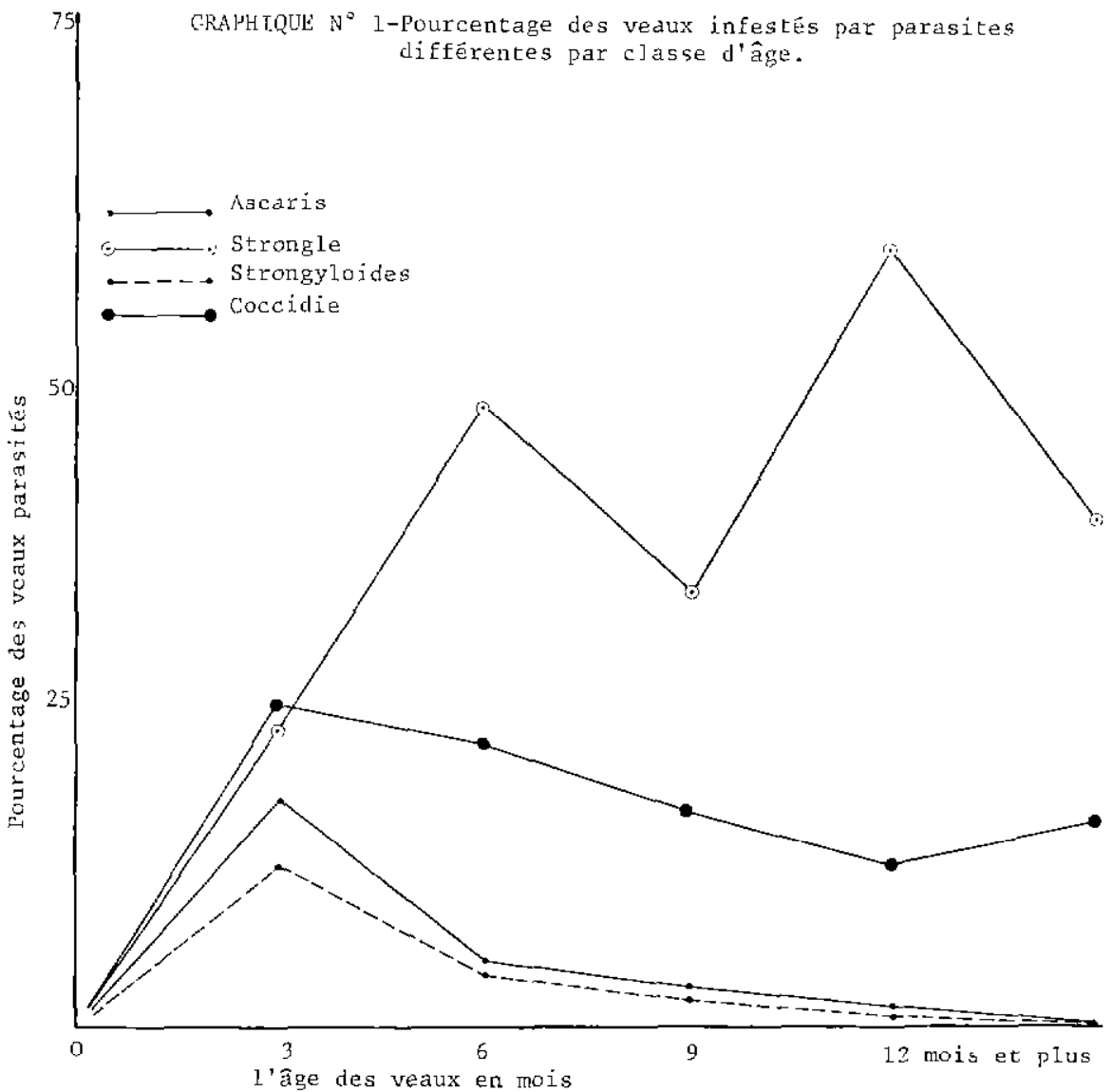

\section{c) Hématoscopies}

a) L'examen de mille neuf cent quarantesept frottis et gouttes épaisses a permis de préciser le taux d'infection par hémoparasite, soit : trypanosomes 14,9 p. 100 ; Babesia 15 p. 100 ; Theileria 6,5 p. 100 ; Anaplasma 2,8 p. 100 et microfilaires 8,6 p. 100 , certains animaux étant par ailleurs porteurs de deux ou plusieurs genres de parasites.

La répartition par espèce et le pourcentage d'infection par rapport au nombre d'animaux reconnus parasités sont : $T$. brucei 54 p. 100 ;
T. vivax 18,6 p. $100 ;$ T. theileri 17,2 p. 100 ; T. congolense 12 p. 100 ; Babesia bovis 55 p. 100 ; B. bigemina 26,7 p. 100 ; Theileria mutans 3 p. 100 et Anaplasma marginale 2,7 p. 100.

Les microfilaires sont certainement de l'espèce Setaria labiato-papillosa car des adultes de cette filaire sont présents dans l'aorte de bovins adultes de la région.

b) Sur mille cinq cent quinze tubes à hématocrites examinés, cent quatre-vingt-onze soit 12,6 p. 100 ont été positifs, les espèces de trypanosomes observées étant les mêmes avec des 

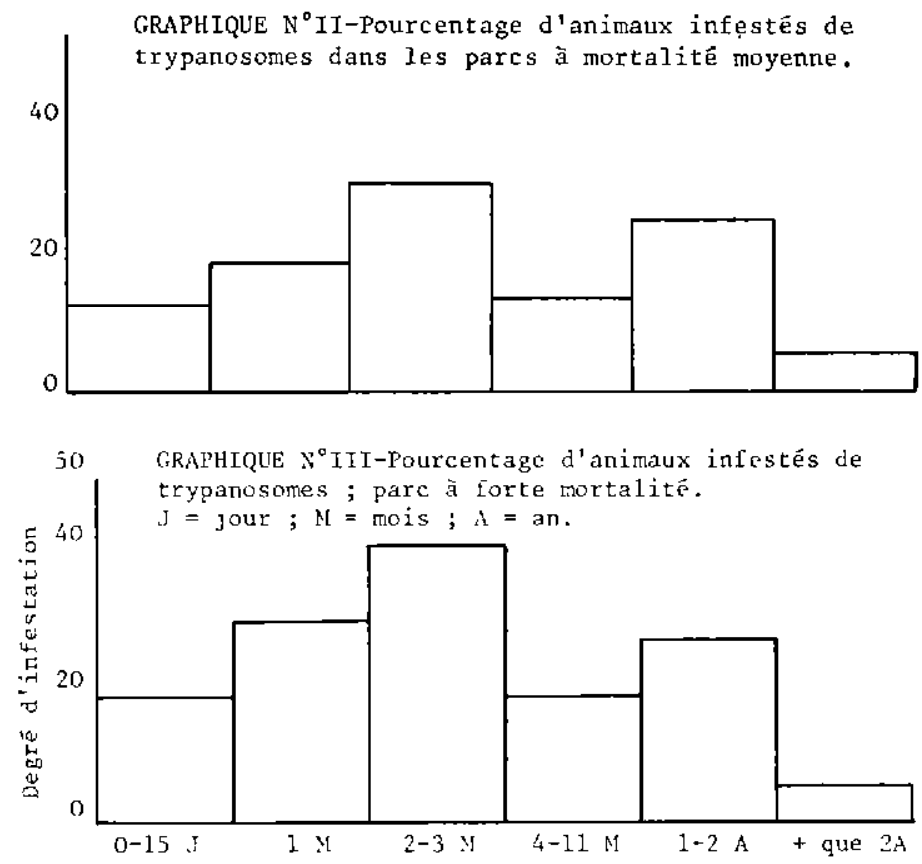

pourcentages d'infection à peu près identiques soit $2 / 3$ de $T$. brucei ; $1 / 4$ de $T$. vivax, le reste étant composé de $T$. congolense et de $T$. theileri.

Il convient de noter qu'en matière de trypanosomes les jeunes animaux, de 0 à 2 ans sont beaucoup plus souvent porteurs de ces hémoparasites : 16,9 p. 100 que les animaux plus âgés : 7,2 p. 100 .

Le graphique II donne la moyenne d'infestation par classe d'âge pour six cent dix-huit animaux entre 0 et 2 ans, dans le cas de parc à mortalité moyenne alors que le graphique III montre que dans les parcs à forte mortalité le taux d'infection des jeunes est nettement plus considérable.

De façon constante la valeur hématocrite des animaux infestés est nettement inférieure à celle des animaux sains, cette différence maximale entre 0 et 1 mois allant par ailleurs en s'atténuant progressivement jusqu'à devenir insignifiante ou presque après l'âge de 2 ans.

\section{Tiques}

Les genres de tiques récoltées ont été par ordre d'importance : Amblyomma ; Boophilus; Hyalomma; Rhipicephalus et Dermacentor.

Leur population varie en fonction de la saison et, comme il est naturel, elle augmente dès le début de la saison sèche (mars) pour atteindre son maximum en juin, pour diminuer rapidement dès l'installation de la saison sèche jusqu'à un minimum qui se situe fin février.
Enfin, dans la seule classe d'âge de 15 jours à 3 mois, il apparaît une différence significative (test de $\mathrm{X} 2$ ) entre les animaux infectés et en mauvais état général par rapport au nombre d'animaux non infectés et en mauvais état général alors qu'à partir de 3 mois révolus la différence observée n'apparaît pas comme significative.

\section{DISCUSSION}

Avant de discuter au vu des résultats de nos observations, il convient de rappeler que O. BREMAUD (2) a signalé que la mortalité des jeunes veaux s'établissait alors entre 35 et 45 p. 100 , dans le nord de la Côte-d'Ivoire et que H. BOHNEL, en 1971 (1), a observé une mortalité élevée (dont il ne donne pas le pourcentage) chez les veaux de 0 à 2 ans dans dix-neuf villages de la région de Korhogo et que, sur cinq cent cinquante-quatre veaux soumis à observation, aucun n'était porteur de trypanosomes, coccidies ou microfilaires.

Le taux de mortalité que nous avons observé est beaucoup plus faible, ce qui paraît tenir à deux causes principales:

- les chiffres donnés par BRÉMAUD ne sont qu'une estimation et l'appréciation de BOHNEL n'intéresse que la région de Korhogo ;

- les progrès constatés peuvent être attribués pour une large part à l'action d'encadrement des éleveurs, qui a démarré en 1972 et s'est poursuivi d'année en année avec des impacts 
progressivement meilleurs et plus prononcés, notamment en matière de trypanosomes et d'hématozoaires, ce qui mérite d'être souligné, alors que l'action pathogène des helminthes est elle-même diminuée du fait que les jeunes animaux sont régulièrement traités, de même en ce qui concerne l'action des tiques.

Le maximum de mortalité se situe en début de saison sèche lorsque les conditions d'alimentation deviennent insuffisantes et concernent des animaux atteints pour la plupart de parasitoses diverses et de gravité variée.

Les différences régionales assez importantes observées dans la mortalité des veaux peuvent s'expliquer, en partie tout au moins, par la répartition des races puisque dans les trois zones où elle est la plus faible $(8,5$ p. 100 à Bouna, Odienné et Touba) ce sont des taurins qui y vivent presque exclusivement : Baoulé à l'Est ; N'Dama à l'Ouest alors que, dans le centre, constitué par les provinces de Korhogo, Boundiali et Ferkéssédougou, les troupeaux sont surtout constitués de métis Baoulé-Zébu. Il faut cependant manier cette observation par le fait que des fortes mortalités, dont les causes n'ont pas été encore déterminées, sont observées dans des troupeaux de Baoulé de la même région.

Des observations recueillies au cours des enquêtes effectuées ont permis de constater que la trypanosomose atteint surtout les jeunes de 0 à 3 mois, qui est bien la classe d'âge la plus infectée dans les parcs à forte mortalité, qui présente les plus fortes différences entre l'hématocrite des animaux porteurs et non porteurs de trypanosomes.

Elle est enfin la seule classe d'âge dont le mauvais état général peut être rattaché à la trypanosomose avec comme conséquence le plus haut taux de mortalité.

A noter que, dans le secteur de Ferkéssédougou, un traitement trypanocide systématique sur des veaux a fait baisser la mortalité de 41 p. 100 à 25 p. 100 .

Les veaux paraissent être infectés dès les premiers jours de leur vie, et même pour certains au travers du placenta puisque 5 veaux ont été trouvés porteurs de trypanosomes, dont deux par $T$. vivax avant leur $15^{\mathrm{e}}$ jour.

La maladie apparaît en général dès le $15^{\mathrm{e}}$ jour avec un taux de mortalité marqué jusqu'au $3^{e}$ mois puis la période critique pour les animaux semble être dépassée ou l'infection diminue alors que l'hématocrite ne varie plus guère et le mauvais état général qui peut alors être constaté ne semble plus être dû aux trypanosomoses.

Par contre et curieusement, le taux d'infection connaît un second pic la deuxième année mais sans paraître affecter la santé de l'animal.

En dehors des symptômes de trypanosomoses, un des symptômes les plus fréquents, dans les parcs à forte mortalité, consiste en une diarrhée sanguinolente que les observations de laboratoire permettent de rattacher d'abord à la coccidiose, ensuite à la strongyloïdose, enfin à un moindre degré à l'ascaridiose, qui est par contre très fréquente chez les animaux traditionnellement élevés.

Les médicaments jusqu'ici utilisés contre le parasitisme intestinal, Tétramisole et Morantel, sont faiblement actifs tant sur les strongyloïdes que les coccidies.

En ce qui concerne les Ixodes chez les animaux régulièrement traités, la moyenne par animal est de 1,33 tique de juillet à octobre et de 0,86 par mois de novembre à mars (7).

Aucun signe clinique n'a jusqu'ici été constaté qui puisse se rapporter à une action pathogène de la part des Babesia, Theileria et Anaplasma malgré les fortes infections constatées.

Ce qui permet de considérer que le rôle des tiques se limite à leur action prédatrice et comme agents de plaies et autres infections cutanées.

Dans cet ordre d'idée, BOHNEL effectuant une enquête d'un mois autour de Korhogo, a constaté, en employant la technique d'agglutination en capillaire l'existence de rickettsies chez les bovins de quatorze villages sur les dix-neuf examinés et des taux très élevés d'Anaplasma : 84,8 p. 100 et de Theileria 98,6 p. 100 , ainsi que l'absence de Boophilus et de Dermacentor parmi les tiques récoltées.

\section{CONCLUSION}

Dans les parcs encadrés, la mortalité des veaux de 0 à 1 an qui s'élève à 11,9 p. 100 et affecte principalement les jeunes sujets âgés de 0 à 3 mois, se situe au début de la saison sèche.

Dans les parcs où le taux de mortalité s'élève à 20 p. 100, ce qui est le cas dans la région de Korhogo, c'est la trypanosomose qui est certainement la cause de cette situation, situation qui peut trouver une explication dans le fait que le cheptel comprend de très nombreux croisements taurin $\times$ zébu. 
La coccidiose et la strongyloïdose sont ensuite et dans l'ordre les principales causes des mortalités observées alors que l'ascaridiose ne semble pas constituer un élément pathogène majeur, contrairement à ce qui est observé dans les parcs non encadrés.

Les tiques ont une action plus indirecte que directe bien que dans certains cas de mortalité précédée de fièvre elles puissent être mises en cause en tani qu'agents vecteurs d'hématozooses diverses.

Pour ce qui est de l'alimentation des veaux, l'étude faite dans deux cent cinquante-quatre parcs a montré qu'il n'existe pas de différence significative entre les mortalités observées dans les parcs où tout le lait de la mère est réservé au veau et ceux où la traite est pratiquée de façon assidue et conséquente, que ce soit pour la consommation de l'éleveur ou la vente.

\section{SUMMARY}

Survey on calf mortality and parasitism in the North of the Ivory Coast :

\section{First observations}

A preliminary survey on parasitism and its effects on calf mortaiity was carried out in the North of the Ivory Coast. The mortality in calves between one day and one year of age was found to average 11,9 p. 100. The mortality was higher at the begining of the dry season November to January principally affecting young calves up to 3 months old.

During the course of this survey work which lasted from January 1976 to June 1977, a large number of faecal samples, blood smears and haematocrite capillary tubes of blood were collected and examined. The major parasitic infestations involved in calf mortality, during the period were found, to be trypanosomiasis, coccidiosis, ascariasis, strongyloidosis and on occasions strongylosis. A part from trypanosomes, the existence of other blood parasites such as Babesia, Theileria, Anaplasma and Microfilariae were recorded which presumably don't seem to play a well defined role in the mortality of calves. In addition, lack of sufficient milk due to milking of cows wherever practised does not seem to be a definite factor for calf-mortality. Effect of seasonal temperature and humidity on the evolution of ticks on the calves body has been studied.

\section{RESUMEN}

\section{Encuesta sobre el parasitismo y la mortalidad de los terneros en el norte de la Costa de Marfil. Observaciones preliminares}

Según las encuestas realizadas en el norte de la Costa de Marfil, la tasa de mortalidad de los terneros llega a 11,9 p. 100. Concierne principalmente los terneros hasta 3 meses de edad y se manifiesta sobretodo al principio de la estación seca, de noviembre a enero. Durante esta encuesta que duró 18 meses (enero 1976-junio 1977), se examinaron numerosas muestras de heces y de sangre, to que permitió establecer que las causas principales de las mortalidades importantes a veces observadas son por orden de importancia las tripanosomo. sis, la coccidiosis, la estrongiloidosis y la estrongilosis mientras que el ascaridiosis ocupa un gran papel en la mortalidad de los terneros en medio de ganaderia tradicional.

Se evidenció la exístencia de Theileria, Babesia, Anaplasma y de microfilarias pero parece que dichos parásitos no desempeñan un papel claramente definido en la mortalidad de los temeros.

Lo mismo, el ordeño de las vacas, donde se práctica no parece intervenir como factor determinante de la mortalidad de los terneros.

\section{BIBLIOGRAPHIE}

1. BOHNEL (H.). Recherche sur des causes de mortalité des veaux dans la savane sous-soudanienne dans le Nord de la Côte-d'Ivoire. Bull. epiz. Dis. afr. 1971, 19 (2) : 145-157.

2. BREMAUD (O.). L'élevage bovin dans le nord de la Côte-d'Ivoire. Paris, SEDES ; Abidjan, Ministère du Plan, 1972.

3. CAMUS (E.). Rapport annuel. Cellule d'appui SODEPRA, Côte-d'Ivoire, 1976.

4. CAMUS (E.). Mortalité des veaux dans les troupeaux encadrés du Nord de la Côte-d'Ivoire. Colloque int. Recherche sur l'Elevage bovin en zone tropicale humide, Bouaké, 18-22 avril 1977.
5. GODET (G.). Cellule d'appui SODEPRA, Korhogo. Communication personnelle.

6. MISHRA (G. S.), Rapport annuel 1976, FAO IVC/71/505. Bingerville, Côte-d'Ivoire, Laboratoire de Pathologie animale, 1977.

7. SODEPRA, Cellule statistique. Récapitulatif par zone des données chiffrées enregistrées de juillet 1976 à mars 1977 dans les parcs encadrés. Côte-d'Ivoire, SODEPRA, 1977.

8. WOO (P, T. K.). The haematocrit centrifuge technique for the diagnosis of african trypanosomiasis. Acto Trop., 1970, $27: 384-386$. 